\title{
Fast-Wave Resonance by Space-Wave Leaky Mode Carrying Dominant-Mode-Like Currents
}

\author{
Ching-Kuang C. Tzuang, Senior Member, IEEE, and Ching-Chyuan Lin
}

\begin{abstract}
By closely examining the resonant phenomena of a suspended, flat wire resonator, this paper speculates the existence of the fast-wave mode causing the anomalous resonance that cannot possibly be explained by the well-known bound mode. Followed by proposing a guiding structure model that allows the assessment of the effects of ground plane and sidewalls (external objects) on the guiding properties, the numerical accuracy of the full-wave spectral-domain approach method for the electrically large model is validated by performing the rigorous convergence study. A series of measurements on the resonator are conducted for the same guiding structure of various lengths, confirming that the anomalous resonance is indeed caused by a fast-wave in the form of a space-wave type leaky mode which also exhibits almost identical modal currents to those of the bound mode.
\end{abstract}

Index Terms-Fast-wave, leaky mode, resonance, space-wave.

\section{INTRODUCTION}

$\mathbf{T}$ THE RESONATOR is a fundamental device to numerous practical designs of microwave circuits and antennas. Coupled-line filters and dipole antennas often employ quarter-wave or half-wave resonators that resonate in a onedimensional fashion [1]-[4]. Furthermore, there are many microwave and antenna circuits incorporating two-dimensional or three-dimensional resonators [5], [6]. This paper reviews the basic properties of the most primitive resonator made of a suspended microstrip and leads to the discovery of the leaky mode which should be combined with the familiar bound mode to constitute a complete modal spectra [7].

Fig. 1 plots the two-port scattering parameters obtained from a properly calibrated vector network analyzer HP8510 which connects its two coaxial connectors' central conductors to both ends of the suspended microstrip of $1.6 \mathrm{~mm}$ in width and $60 \mathrm{~mm}$ in length. Since there is nothing other than the air nearby the suspended microstrip, a bound mode of phase constant $\beta_{b}$ nearly the same as the free-space wave number $k_{0}$ $\left(2 \pi / \lambda_{0} ; \lambda_{0}\right.$ is the free-space wavelength) should be present in the transmission line. If this bound mode would cause resonance, and no coupling between the input and output ports are assumed, the resonant frequencies can be estimated by the relation

$$
\beta_{b} \times l=n \pi
$$

Manuscript received March 30, 1998; revised September 3, 1998. This work was supported by the National Science Council, Taiwan, under Grant NSC 87-2213-E-009-105 and Grant NSC 87-2213-E-009-106.

The authors are with the Institute of Electrical Communication Engineering, National Chiao Tung University, Hsinchu, Taiwan.

Publisher Item Identifier S 0018-9480(98)09189-3.

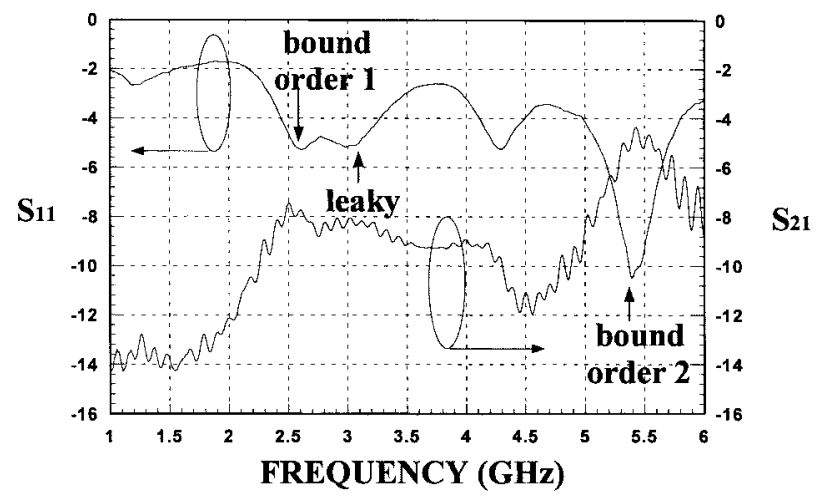

Fig. 1. The two-port scattering parameters obtained from the HP8510 for a suspended microstrip in the air of $60 \mathrm{~mm}$ in length and $1.6 \mathrm{~mm}$ in width. The suspended microstrip is a bare wire without any support by dielectric substrate.

where $n$ is a positive integer, representing the order of resonance; $l$ is the length of the suspended line. Equation (1) mandates that constructive phase condition be maintained to sustain the resonance for an electromagnetic wave returning to the launching position after multiple reflections at both ends of the suspended microstrip.

Let us further denote the normalized phase constant of the bound mode as

$$
\hat{\beta}_{b}=\frac{\beta_{b}}{k_{0}} .
$$

Combining (1) and (2), we obtain the bound-mode resonant frequency in $f_{b_{-} r e s}$ of order $n$ as follows:

$$
f_{b \_r e s}=\frac{n}{2} \frac{1}{\hat{\beta}_{b}} \frac{c}{l}
$$

where $c$ is the speed of light.

The measured resonant frequency of the lowest order bound mode at $2.613 \mathrm{GHz}$ can be predicted very accurately by substituting $n=1, \hat{\beta}_{b}=1$, and $l=60 \mathrm{~mm}$ to (3). So is the measured second bound-mode resonance at $5.4 \mathrm{GHz}$ by letting $n=2$. What puzzles us is the adjacent resonance at 3.063 $\mathrm{GHz}$ to the right of the lowest order resonant frequency. If we assume that there is another lowest order resonance caused by an additional mode, we may conclude immediately that the normalized phase constant of the new mode must be smaller than one, so that its resonance frequency is higher than that of the bound mode. This manifests the so-called fast-wave resonance since its phase velocity is greater than the speed of light.

This paper aims to validate the assumption, explore and discuss the guiding properties of the additional mode which 


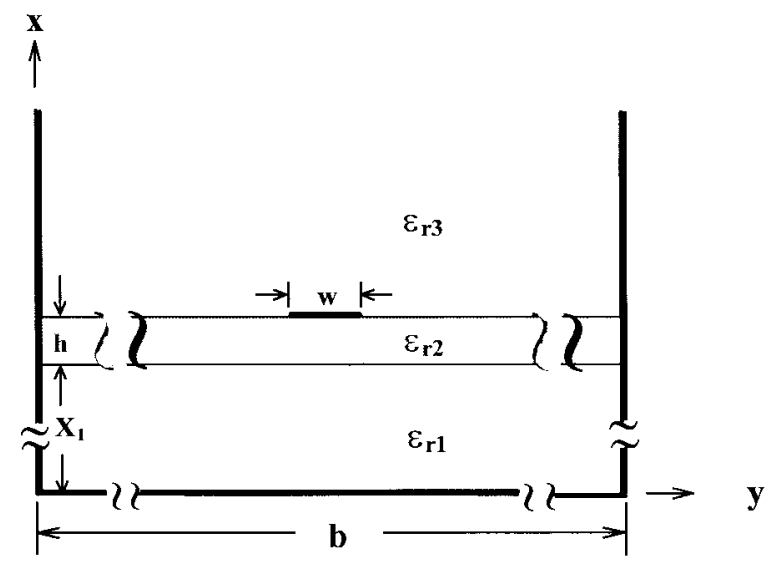

Fig. 2. The cross-sectional model of the suspended wire resonator whose test results are partly shown in Fig. 1. The parameters of the guiding model are $\varepsilon_{r l}=\varepsilon_{r 2}=\varepsilon_{r 3}=1.0, h=0.762 \mathrm{~mm}, x_{1}=1000 \mathrm{~mm}, b=11 \lambda_{0} \mathrm{~mm}$.

turns out to be a space-wave type leaky mode carrying dominant-mode-like current distributions on the suspended microstrip. Section II describes the model and method for obtaining the guiding properties of the new leaky mode discussed in detail in Section III. Section IV returns to the resonant phenomena of the suspended wire mentioned above and investigates the joint contributions to the resonances out of the bound mode and the leaky mode. Section V concludes the paper.

\section{Model AND Method OF ANALYSIS}

The speculation of the additional mode contributing to the unknown cause for the resonance that cannot possibly be explained by the bound modes leads to a proposed guiding structure model as shown in Fig. 2 to be explained as follows.

To obtain the scattering parameters of the suspended microstrip immersed in the air, the transmission line can hardly isolate itself from the surrounding objects since the vector network analyzer (VNA) usually does not allow excessive length of cables from the measurement ports. Thus, we propose a simple model that contains a suspended microstrip placed above a ground plane and in the middle of a two parallel plates. The model will permit us to assess the effects of the ground plane as well as the sidewalls on the modal properties of the microstrip which can be either in the air (by setting $\epsilon_{r}=1.0$ ) or on a substrate. These two cases are investigated thoroughly in the paper.

The formulation employed for tackling the guided-wave problem depicted in Fig. 2 is the spectral-domain approach (SDA) [8] outlined in detail elsewhere [9]. The Galerkin's numerical algorithm together with the subdomain triangular basis functions are adopted for computing the complex propagation constant $\gamma=\alpha+j \beta$, where $\alpha$ is the attenuation constant and $\beta$ the phase constant. Throughout the paper the propagation constant, either purely imaginary for a strictly bound mode or complex for a leaky mode, is normalized to $k_{0}$, the free-space wave number $\left(2 \pi / \lambda_{0}\right)$.

The sidewalls separation distance $b$ and the suspension height $x_{1}$ must be kept at a far enough distance if one desires to keep their influences on the guided-mode propagation minimal.
Since Fig. 2 essentially models the suspended microstrip in a U-shaped electric container, which can by no means excite the surface wave, the modes that we will obtain must be either bound modes or leaky modes [10]. The former had been extensively studied before [11], i.e., the even mode with nonzero cutoff dispersion characteristics. The latter will be shown to cause the space-wave resonance as speculated in the introduction section. One should notice that if the leaky mode exists in the model depicted in Fig. 2, the leakage or the radiation must take place in the form of space wave only as the possibility of exciting the surface wave is totally eliminated [12]. Therefore, the value of the normalized phase constant of this leaky mode should be smaller than one to incur space-wave radiation.

\section{NUMERICAL RESULTS}

Before presenting the solutions for the bound mode and the leaky mode of the suspended microstrip modeled in Fig. 2, the numerical accuracy must be confirmed. The validity of the SDA employed in the paper will be established by obtaining the accuracy criteria for 1) the number of subdomain bases and harmonics as needed in the SDA formulation to have the converged values for the complex propagation constants, 2 ) the minimal suspended heights $\left(X_{1}\right)$ required to prevent the ground plane from influencing the complex propagation constants of the leaky mode, and 3) the separation distance (b) of the parallel plates that produce very little influence on the complex propagation constant.

\section{A. Convergence Study}

Let $p$ be the positive integer representing the number of the subdomain bases applied to expand the SDA modal currents in both longitudinal and transverse directions of the suspended microstrip. Thus, the physical width of the triangular subdomain basis is $w / p$, where $w$ is the width of microstrip. The total number of the harmonics $\left(M_{r}\right)$ employed in the SDA is

$$
M_{r}=k \frac{b}{w / p}
$$

where $k$ is a scaling factor and was applied in [13]. Applying the structure parameters and the material constants as shown in Fig. 3, we plot a leaky-mode solution at $2.75 \mathrm{GHz}$ using the normalized propagation constant against the scaling factor $k$. Parts (a) and (b) of Fig. 3 plot the normalized phase constant and attenuation constant, respectively. Accordingly, when $k=1, M_{r}=6300$ for $p=24, b=420 \mathrm{~mm}$, and $w=1.6 \mathrm{~mm}$. In Fig. 3, $k$ and the three different values of $p, 24,32$, and 56, are used as the controlling parameters. As shown in Fig. 3(a), the normalized phase constant $\left(\beta / k_{0}\right)$ converges to 0.708915 for $k \geq 1$, regardless of the values of $p$. When $p=24(56)$, the normalized phase constant $\left(\beta / k_{0}\right)$ converges to error $\left(\left(\beta_{M r}-\beta_{M r-1}\right) / \beta_{M r}\right)$ of $6.97 \times 10^{-6}$ $\left(3.82 \times 10^{-6}\right), 2.34 \times 10^{-6}\left(1.14 \times 10^{-6}\right)$ and $1.06 \times$ $10^{-6}\left(4.94 \times 10^{-7}\right)$ for $k=1,1.5$, and 2 , respectively. On the other hand, the convergence properties of the normalized attenuation constant $\left(\alpha / k_{0}\right)$ are slightly different. The values of $p$ have relatively small influences on the converged values. 


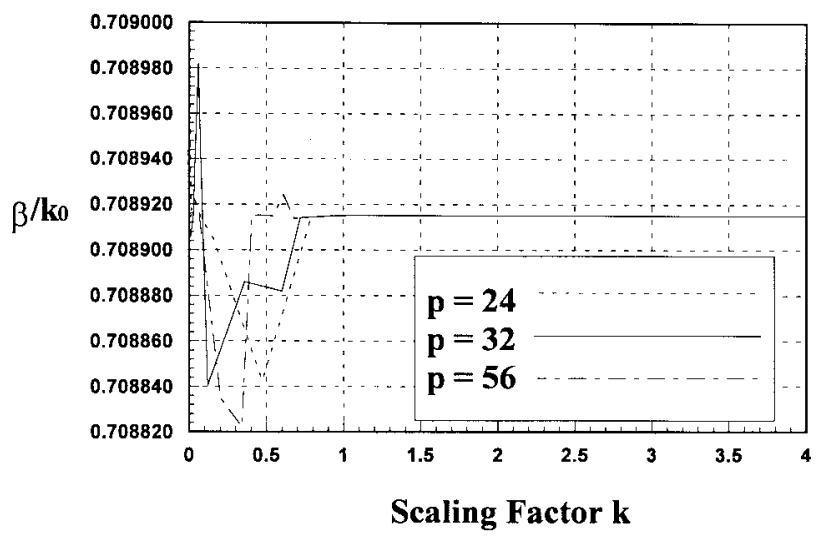

(a)

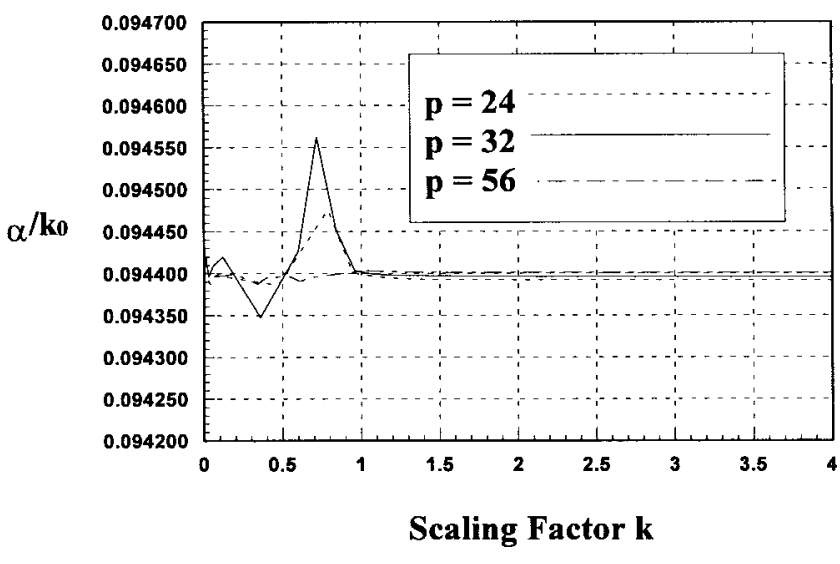

(b)

Fig. 3. The convergence properties of the SDA for computing the complex propagation constant of the leaky mode at $2.75 \mathrm{GHz}$ on the suspended microstrip with parameters $b=420 \mathrm{~mm}, x_{1}=300 \mathrm{~mm}, w=1.6 \mathrm{~mm}$, $h=0.762 \mathrm{~mm}, \varepsilon_{r 1}=\varepsilon_{r 3}=1.0 . p$ : the number of triangular bases used for representing the currents on the strip, $k$ : the scaling factor defined by (4): (a) the normalized phase constant and (b) the normalized attenuation constant.

When $p=24(56)$, the normalized attenuation constant $\left(\alpha / k_{0}\right)$ converges to error $\left(\left(\alpha_{M r}-\alpha_{M r-1}\right) / \alpha_{M r}\right)$ of $4.53 \times 10^{-3}(2.61$ $\left.\times 10^{-3}\right), 1.50 \times 10^{-3}\left(7.71 \times 10^{-4}\right)$ and $6.82 \times 10^{-4}(3.35 \times$ $10^{-4}$ ) for $k=1,1.5$, and 2 , respectively. Based on the results shown in Fig. 3(a) and (b), we choose $k=2$ and $p=24$ for obtaining all the numerical data following Fig. 3, with confidence that the numerical accuracy is much better than the experimental tolerance. Fig. 3 shows the absolute convergence properties that are similar to those reported in [14], where the converged solutions were obtained by a sufficiently large number of satisfactory basis functions together with a large inner product truncation point.

\section{B. Effects of Ground Plane Distance $x_{1}$}

By placing the sidewalls at a far enough distance and varying the height of the suspended microstrip, Fig. 4 plots the normalized phase and attenuation constants against $x_{1}$ normalized to the free-space wavelength $\lambda_{0}$. The operating frequency is chosen as $3.0 \mathrm{GHz}$. Two types of modes are observed. One is the bound mode which is the well-known suspended microstrip mode of $\beta / k_{0}$ approaching 1.062 for $x_{1} / \lambda_{0}$ approximately equal to $0.5 \lambda_{0}$. As $x_{1} / \lambda_{0}$ is reduced

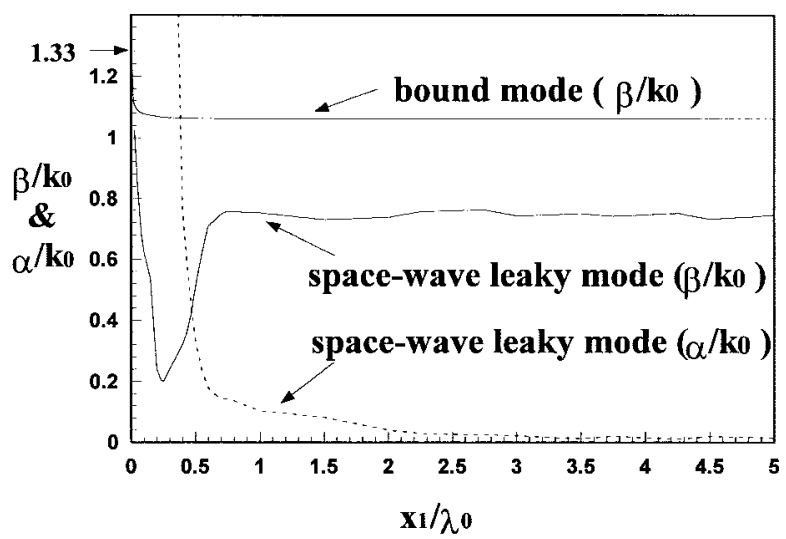

Fig. 4. Effects of the ground plane distance on the leaky mode propagating on the suspended microstrip with substrate permittivity of $\varepsilon_{r 2} \varepsilon_{0}$. Parameters of the suspended microstrip: $b=1000 \mathrm{~mm}, w=1.6 \mathrm{~mm}, h=0.762 \mathrm{~mm}$, $\varepsilon_{r 2}=2.1, \varepsilon_{r 1}=\varepsilon_{r 3}=1.0$, and $f=3.0 \mathrm{GHz}$.

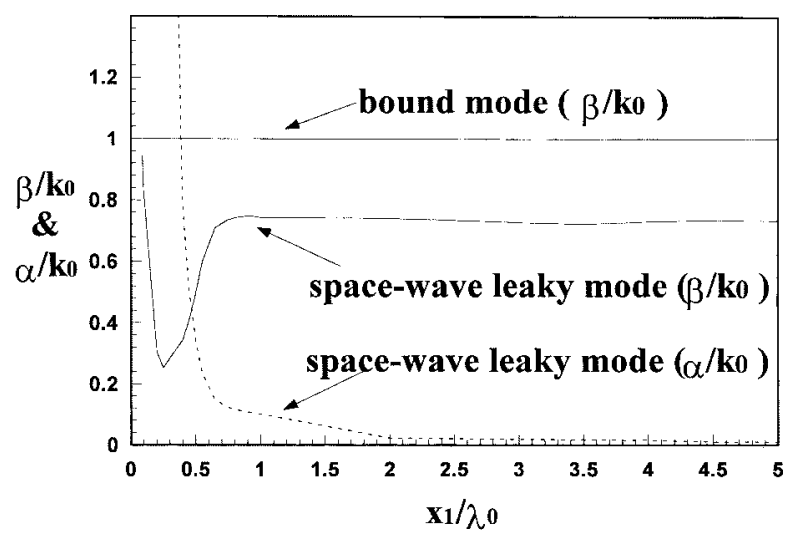

Fig. 5. Effects of ground plane distance on the leaky mode propagating on the suspended microstrip without substrate. Parameters of the suspended microstrip: $b=1000 \mathrm{~mm}, w=1.6 \mathrm{~mm}, \varepsilon_{r 1}=1.0, \varepsilon_{r 1}=\varepsilon_{r 3}=1.0$, and $f=3.0 \mathrm{GHz}$.

to zero, the $\beta / k_{0}$ is identical to that of microstrip mode, i.e., 1.33. Leaky mode changes substantially for $x_{1}$ smaller than $0.75 \lambda_{0}$. As $x_{1}$ increases from $0.75 \lambda_{0}$, the $\beta / k_{0}$ soon reaches to a constant value. The $\alpha / k_{0}$ increases to a large value when $x_{1}<0.75 \lambda_{0}$ and reaches a constant value when $x_{1}>2.5 \lambda_{0}$. Therefore, for the similar guiding structure under investigation, we choose $x_{1}$ equal to $3 \lambda_{0}$ to simulate the test environment in which the ground plane has little influence on the leaky mode propagation characteristics.

By setting $\varepsilon_{r}$ equal to 1.0 , simulating the test condition of the same microstrip surrounded by the air only, we observe in Fig. 5, as expected, the normalized phase constant of the bound mode equal to 1.0, independent of the variation of $x_{1}$. The leaky mode's complex propagation constants are nearly the same as the previous case.

\section{Effects of Sidewalls Distance b}

Using the same suspended microstrip structure and setting the ground plane distance $x_{1}$ equal to $1000 \mathrm{~mm}$ (equivalent to $3.33 \lambda_{0}$ at $1 \mathrm{GHz}$ ), we plot in Fig. 6 the normalized phase and attenuation constants of the new leaky mode versus frequencies between 1 and $5 \mathrm{GHz}$ by varying the sidewalls' distance 


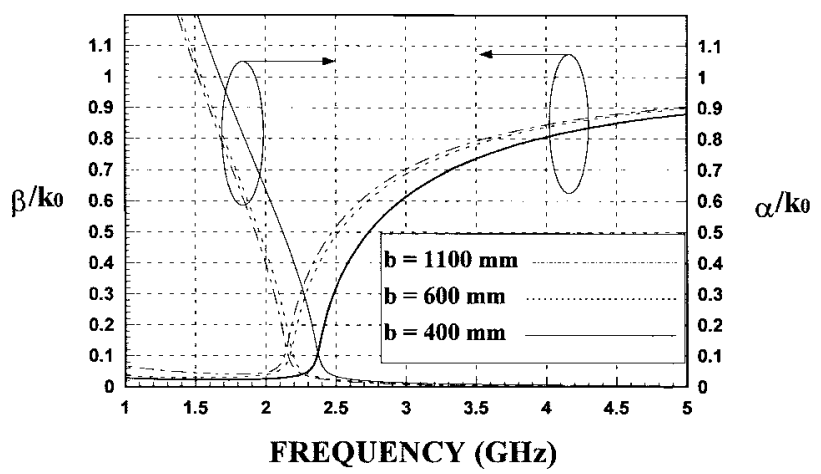

Fig. 6. Effects of the sidewalls on the leaky mode propagating on the suspended microstrip. Parameters of the suspended microstrip: $x_{1}=1000$ $\mathrm{mm}, w=1.6 \mathrm{~mm}, h=0.762 \mathrm{~mm}, \varepsilon_{r 2}=2.1$, and $\varepsilon_{r 1}=\varepsilon_{r 3}=1.0$.

$b$ from $400 \mathrm{~mm}$, through $600 \mathrm{~mm}$, to $1100 \mathrm{~mm}$, respectively. The solutions for the complex propagation constant converge quickly as $b$ is increased from 400 to $1100 \mathrm{~mm}$ as depicted in Fig. 6. The deviations in the dispersion characteristics between the cases for $b=600 \mathrm{~mm}$ and $b=1100 \mathrm{~mm}$ are less for frequencies above $3 \mathrm{GHz}$ than below $3 \mathrm{GHz}$. At $3 \mathrm{GHz}$, the free-space wavelength is $100 \mathrm{~mm}$ and $b$ of $1100 \mathrm{~mm}$ is equal to $11 \lambda_{0}$. Thus, the sidewalls will have very little influence on the dispersion characteristics of the leaky mode if the walls are kept approximately $5.5 \lambda_{0}\left(11 \lambda_{0} / 2\right)$ away from the suspended line.

\section{Effects of Symmetry}

In the case when the sidewalls are truly at a far away distance, the exact location of the suspended microstrip must be immaterial. In other words, the obtained complex propagation constants should be insensitive to the symmetry of the guiding structure. We perform the test on the symmetry of the suspended microstrip by moving the central strip $1 \mathrm{w}$ and $10 \mathrm{w}$ away from the center of the guide. During the numerical computations, the distance between two sidewalls are always scaled to $11 \lambda_{0}$ when frequency is changing. The results are plotted in Fig. 7, comparing the effects of symmetry on the leaky mode's complex propagation constants. The symmetric line and the two offset lines display almost identical dispersion characteristics, revealing that the offset has virtually negligible effects on the leaky-mode propagation characteristics.

Fig. 8 plots the normalized modal currents of the suspended line offset by $10 \mathrm{w}(16 \mathrm{~mm})$ from center for both bound mode and leaky mode, respectively. Notice that both longitudinal and transverse portions of the two modes are nearly the same. Furthermore, comparing the data shown in Fig. 8 with those of [12, Fig. 3], where the centered, symmetric suspended microstrip was investigated, the modal currents are nearly identical. Thus, the leaky mode's propagation characteristics are insensitive to the external objects kept at a distance approximately $5.5 \lambda_{0}$ away from the suspended microstrip.

\section{Wire Resonator And Space-WaVe Leaky Mode}

Similarities of bound mode and leaky mode in modal current distributions (see Fig. 8) and transverse electromagnetic field

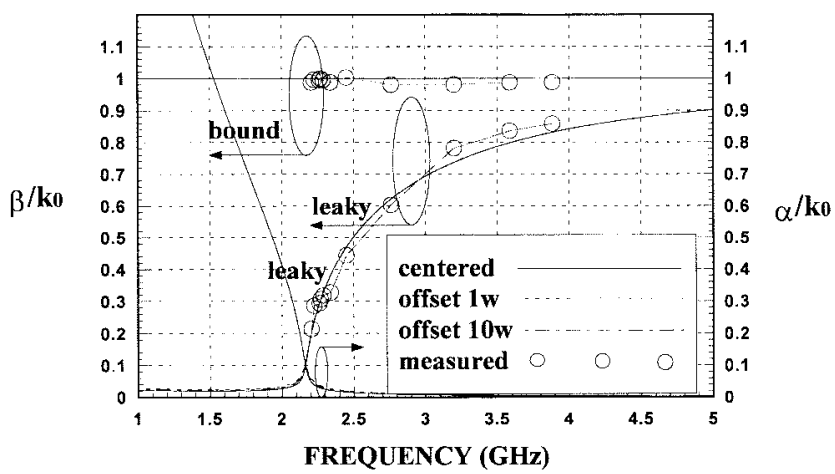

Fig. 7. The dispersion characteristics of the bound mode and the leaky mode of the various suspended microstrips without supporting substrate. The parameters of the guiding structure: $b=11 \lambda_{0}$ (normalized to the operating frequency), $x_{1}=1000 \mathrm{~mm}, w=1.6 \mathrm{~mm}, h=0.762 \mathrm{~mm}, \varepsilon_{r 2}=1.0$, $\varepsilon_{r 1}=\varepsilon_{r 3}=1.0$. One symmetric line and two offset lines by $1 \mathrm{w}$ and 10 $\mathrm{w}$ offsets from center are investigated. $(w=1.6 \mathrm{~mm})$. The circle symbols represent the estimated values for the phase constants of the bound mode and leaky mode whose resonant frequencies are measured and tabulated in Table I.

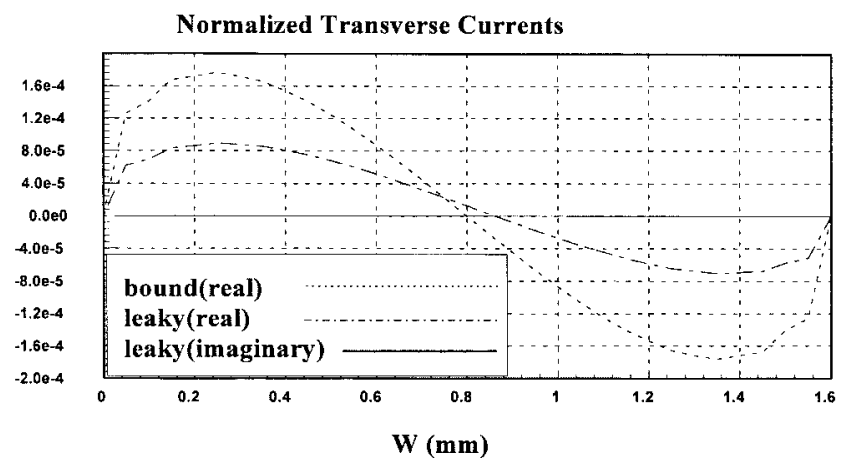

(a)

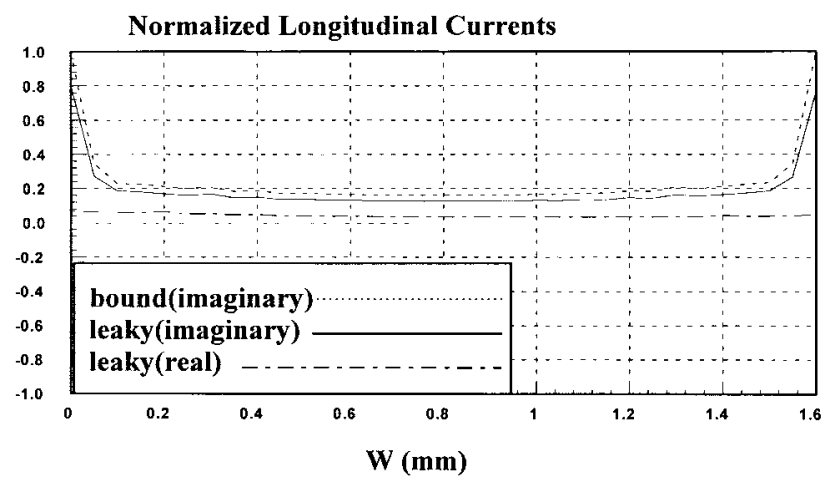

(b)

Fig. 8. Normalized current distribution of the asymmetric suspended microstrip located off center of $10 \mathrm{w}(16 \mathrm{~mm})$ : (a) the normalized transverse currents of bound and leaky modes and (b) the normalized longitudinal currents of bound and leaky modes. Operating frequency: $2.75 \mathrm{GHz}$.

distributions surrounding strip (see [12, Figs. 4 and 5]) are amenable to our speculation of the existence of the additional fast-wave mode. Fig. 7 is a clear manifestation of the leaky mode which is in addition to the bound mode while exhibiting a fast-wave mode type. As the suspended microstrip either analyzed in Fig. 7 or reported in Fig. 1 are the same guiding structure, we compute the first resonant frequency of the leaky mode for the suspended microstrip of $60 \mathrm{~mm}$ (shown in Fig. 1) 
TABLE I

Resonant Frequencies ObTained by MEasurements and THEORIES

\begin{tabular}{|c|c|c|c|c|c|c|c|}
\hline length & leaky & $\begin{array}{l}\text { bound } \\
\text { order } 1\end{array}$ & $\begin{array}{l}\text { bound } \\
\text { order } 2\end{array}$ & $\begin{array}{l}\text { bound } \\
\text { order } 3\end{array}$ & $\begin{array}{l}\text { bound } \\
\text { order } 4\end{array}$ & $\begin{array}{c}\text { bound } \\
\text { order } 5\end{array}$ & $\begin{array}{c}\text { bound } \\
\text { order } 6\end{array}$ \\
\hline \multirow{2}{*}{$l$} & measured & measured & measured & measured & measured & measured & measured \\
\hline & predicted & prcdicted & predicted & predicted & predicted & predicted & predicted \\
\hline \multirow{2}{*}{45} & 3.885 & 3.253 & & & \multirow{2}{*}{\multicolumn{3}{|c|}{ unit : length $l: \mathrm{mm}$}} \\
\hline & $\overline{3.970}$ & $\overline{3.333}$ & & & & & \\
\hline \multirow{2}{*}{50} & 3.488 & 2.944 & & & \multirow{2}{*}{\multicolumn{3}{|c|}{ resonant frequency: $\mathrm{GHz}$}} \\
\hline & 3.697 & $\overline{3.000}$ & & & & & \\
\hline \multirow{2}{*}{60} & 3.063 & 2.613 & & & & & \\
\hline & $\overline{3.310}$ & $\overline{2.500}$ & & & & & \\
\hline \multirow{2}{*}{70} & 2.760 & $N A$ & & & & & \\
\hline & 3.040 & 2.140 & & & & & \\
\hline \multirow{2}{*}{90} & 2.396 & $N A$ & 3.294 & & & & \\
\hline & 2.720 & 1.667 & 3.333 & & & & \\
\hline \multirow{2}{*}{138} & 2.421 & $N A$ & $N A$ & 3.273 & & & \\
\hline & 2.480 & 1.087 & 2.174 & 3.261 & & & \\
\hline \multirow{2}{*}{196} & 2.356 & $N A$ & $N A$ & $N A$ & 3.075 & 3.763 & \\
\hline & 2.293 & $\overline{0.765}$ & $\overline{1.531}$ & $\overline{2.296}$ & $\overline{3.061}$ & $\overline{3.825}$ & \\
\hline \multirow{2}{*}{206} & 2.338 & NA & $N A$ & $N A$ & 2.938 & 3.581 & \\
\hline & 2.280 & $\overline{0.728}$ & $\overline{1.456}$ & $\overline{2.184}$ & $\overline{2.913}$ & $\overline{3.641}$ & \\
\hline \multirow{2}{*}{216} & $\underline{2.275}$ & $N A$ & NA & $N A$ & 2.868 & $\underline{3.500}$ & \\
\hline & 2.270 & $\overline{0.694}$ & $\overline{1.389}$ & $\overline{2.083}$ & $\overline{2.778}$ & $\overline{3.472}$ & \\
\hline \multirow{2}{*}{226} & $\underline{2.250}$ & $N A$ & $N A$ & $N A$ & 2.744 & 3.294 & 4.000 \\
\hline & $\overline{2.263}$ & $\overline{0.664}$ & $\overline{1.327}$ & $\overline{1.991}$ & $\overline{2.655}$ & $\overline{3.319}$ & $\overline{3.983}$ \\
\hline \multirow{2}{*}{236} & $\underline{2.200}$ & $N A$ & $N A$ & $N A$ & 2.669 & 3.194 & 3.900 \\
\hline & $\overline{2.253}$ & $\overline{0.636}$ & $\overline{1.271}$ & $\overline{1.907}$ & 2.542 & 3.178 & $\overline{3.816}$ \\
\hline \multirow{2}{*}{318} & 2.050 & $N A$ & $N A$ & $N A$ & $N A$ & 2.394 & 2.869 \\
\hline & 2.213 & 0.472 & 0.943 & 1.415 & 1.887 & 2.358 & 2.830 \\
\hline
\end{tabular}

$\mathrm{NA}$ : The resonant frequencies are not apparent from the measurements.

in length using the following equation:

$$
f_{l-\text { res }}=\frac{n}{2} \frac{1}{\hat{\beta}_{l}} \frac{c}{l} .
$$

Equations (3) and (5) are essentially the same except the types of mode employed in the analysis. Looking up the value of $\hat{\beta}_{l}$ of Fig. 7 at the first, additional resonance frequency at $3.063 \mathrm{GHz}$ and substituting $\hat{\beta}_{l}$ into (5), we obtain the resonant frequency of the new, additional leaky mode at $f_{l-\text { res }}=3.310$ $\mathrm{GHz}$, which is $7.46 \%$ away from the measured value. Thus, the link between the anomalous resonance of the wire and the fast-wave assumption is established by the newly found leaky mode which is certainly a fast wave, causing the radiation into space.

Maintaining the same guiding structure and varying the length of the suspended microstrip for $l=45,50,60,70,90$, $138,196,206,216,226,236,318 \mathrm{~mm}$, respectively, we measured the resonant frequencies for the leaky mode and the bound mode and tabulated them in Table I, where the measured and predicted resonant frequencies are stacked for comparison. In the first column of Table I both measured and predicted resonant frequencies caused by the leaky mode agree well, $12 \%$ for the worst and $0.22 \%$ for the best. As moving toward the lower frequency end, the vast change of the phase constant of the leaky mode (see Fig. 7) is compensated by the increased length of up to six times and more, rendering a mild reduction in resonance frequency from 3.885 to $2.050 \mathrm{GHz}$.

Contrary to the leaky mode, the bound mode's normalized phase constant is very close to one throughout the interested frequencies, thereby the resonant frequency of the bound mode

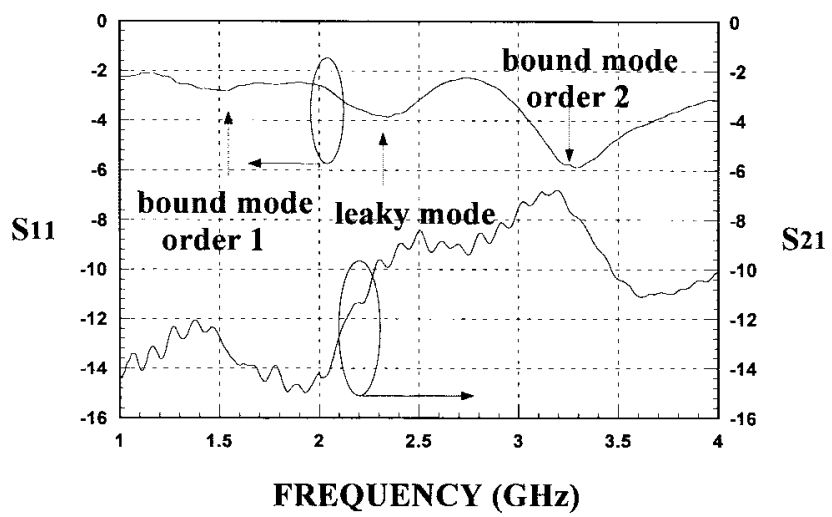

Fig. 9. Typical resonant phenomena of a longer suspended microstrip without supporting substrate. The parameters of the guiding structure are the same as those for Figs. 1, 7, and 8 except the length $l$ is $90 \mathrm{~mm}$.

is inversely proportional to the length $l$ of the guide. As the length $l$ is increased from 45 to $318 \mathrm{~mm}$ (more than six times) we expect the bound mode resonance of order six should enter the frequency spectrum of interest. Such expectation is observed in Table I where the bound mode resonance of order six does come into the spectrum for $l$ greater than 226 $\mathrm{mm}$. When $l$ is increased, the resonant frequencies of both bound mode and leaky mode move to the lower frequency end. However, the bound mode moves at a faster rate because of the above-mentioned reasons, causing the resonant frequency of the bound mode of higher orders to move past that of the leaky mode. Fig. 9 records the resonant phenomena of the same suspended microstrip of length equal to $90 \mathrm{~mm}$, illustrating the movement of the resonant frequencies. Fig. 9 shows that the first bound mode's resonance is much less visible than its apparent visibility in Fig. 1, while at the same time the second bound mode's resonance approaches in. Thus we observe that the bound mode's first resonance is much less visible than those of the leaky mode and the bound mode of order two (or higher). Correspondingly, Table I shows the notion of NA (not apparent) at the fifth row $(l=90 \mathrm{~mm})$ and third column (bound mode of order 1). As length $l$ continues increasing, more and more higher order resonances move left, past the leaky mode resonance, and become nearly invisible. Therefore, the notion of NA in Table I reflects the disappearing of these resonant phenomena of lower order in the measurements.

Conversely we may estimate the leaky mode's phase constant by substituting the measured resonant frequencies of the leaky mode together with the corresponding lengths into (5). The results are plotted in Fig. 7, where the measured values denoted by the circle symbols agree very well with the theoretical data. The fast-wave resonance of the suspended microstrip in the air is indeed the result of the space-wave leaky mode propagating back and forth in the wire.

\section{CONCLUSION}

The discovery of the space-wave type leaky mode is confirmed experimentally and theoretically by proving its presence at a suspended microstrip resonator which shows fast-wave resonance. 
Using the fast-wave resonant frequencies measured at various lengths of the wire resonator, we estimate the phase constant of the leaky mode in very good agreement with the full-wave SDA solutions. Conversely, the anomalous resonant frequencies which are clearly not from the bound mode can be estimated very accurately by applying the dispersion characteristics of the new leaky mode. Detailed analyses show that the space-wave leaky mode carries dominant-mode-like currents, implying that the suspended microstrip, with or without substrate support, is basically a multimode structure subject to potentially significant radiation. The research for the space-wave leaky mode is at its infant stage; the authors expect more investigations will be conducted in this interesting field.

\section{REFERENCES}

[1] G. L. Matthaei, L. Young, and E. M. T. Jones, Microwave Filters, Impedance-Matching Networks, and Coupling Structure. Dedham, MA: Artech, 1980, ch. 8-10.

[2] G. L. Matthaei and G. L. Hey-Shipton, "Concerning the use of hightemperature superconductivity in planar microwave filters," IEEE Trans. Microwave Theory Tech., vol. 42, pp. 1287-1293, July 1994

[3] Y. T. Lo and S. W. Lee, Antenna Handbook. New York: Van Nostrand Reinhold, 1988, ch. 23-26.

[4] V. Trainotti, "MF AM asymmetric vertical-dipole-antenna measurements," IEEE Trans. Antennas Propagat. Mag., vol. 38, pp. 75-82, Dec. 1996.

[5] K. C. Gupta, R. Garg, and R. Chadha, Computer-Aided Design of Microwave Circuits. Dedham, MA: Artech, 1981, ch. 8.

[6] R. K. Hoffmann, Handbook of Microwave Integrated Circuits. Norwood, MA: Artech, 1987.

[7] N. Marcuvitz, "On field representations in terms of leaky modes or eigenmodes," IRE Trans. Antennas Propagat., vol. AP-4, pp. 192-194, July 1956.

[8] T. Itoh, "Spectral domain immitance approach for dispersion characteristics of generalized printed transmission lines," IEEE Trans. Microwave Theory Tech., vol. MTT-28, pp. 733-736, July 1980.

[9] G.-J. Chou and C.-K. C. Tzuang, "An integrated quasiplanar leaky-wave antenna," IEEE Trans. Antennas Propagat., vol. 44, pp. 1078-1085, Aug. 1996.

[10] K.-F. Fuh and C.-K. C. Tzuang, "The effects of covering on complex wave propagation in gyro magnetic slot lines," IEEE Trans. Microwave Theory Tech., vol. 43, pp. 1100-1105, May 1995.

[11] R. H. Jansen, "Unified user-oriented computation of shield, covered and open planar microwave and millimeter-wave transmission-line,"
Microwaves, Optics Acoust., vol. 3, no. 1, pp. 14-22, Jan. 1979.

[12] C.-K. C. Tzuang and C.-C. Lin, "Space-wave-type leaky mode carrying dominant-mode-like current distributions," in IEEE MTTT-S Int. Microwave Symp. Dig., June 1998, vol. 2, Section WE3A-1, pp. 643-646.

[13] L. P. Dunleavy and P. B. Katehi, "A generalized method for analyzing shield thin microstrip discontinuities," IEEE Trans. Microwave Theory Tech., vol. MTT-36, pp. 1758-1766, Dec. 1988.

[14] K. J. Webb and P. W. Grounds, "Convergence in the spectral domain formulation of waveguide and scattering problems," IEEE Trans. Antennas Propagat., vol. 38, pp. 869-877, June 1990.

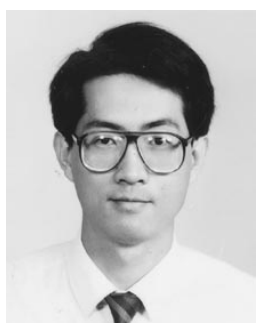

Ching-Kuang C. Tzuang (S'84-M'86-SM'92) was born in Taiwan, R.O.C., on May 10, 1955. He received the B.S. degree in electronic engineering from the National Chiao Tung University, Hsinchu, Taiwan in 1977, the M.S. degree from the University of California, Los Angeles in 1980, and the Ph.D. degree in electrical engineering from the University of Texas at Austin in 1986

From February 1981 to June 1984, he was with TRW, Redondo Beach, CA, working on analog and digital monolithic microwave integrated circuits. While at the University of Texas at Austin, he worked on high-speed transien analyses of monolithic microwave integrated circuits. Since September 1986, he has been with the Institute of Communication Engineering, National Chiao Tung University, Hsinchu, Taiwan. His research activities involve the design and development of millimeter-wave and microwave active and passive circuits and the field theory analysis and design of various quasi-optical integrated antennas.

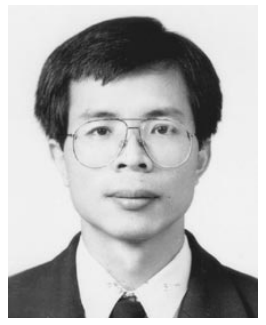

Ching-Chyuan Lin was born in Taiwan on November 7,1958 . He received the B.S. degree in physics from the Soochow University, Taipei, Taiwan, in 1982 and the M.S. degree in space science from the National Central University, Chung-Li, Taiwan, in 1984 . He is currently working toward the Ph.D. degree at National Chiao Tung University in communication engineering.

From 1984 to 1994 , he was with the Chung Shang Institute of Science and Technology, LungTan, Taiwan. His current research interests include field theory analysis and its application on designing leaky mode antennas. 